\title{
Effect of Exercise on Reported Physical Sexual Satisfaction of University Students
}

\author{
Holly C. Lindeman, Keith A. King, Bradley R. Wilson \\ University of Cincinnati
}

\begin{abstract}
The purpose of this study was to examine sexually active university students' reported level of physical sexual satisfaction and perceived impact of exercise on physical satisfaction. In addition, this study investigated whether reported physical sexual satisfaction and perceived impact of exercise on physical sexual satisfaction differed based on current exercise status, body mass index, and gender. A sample of 678 college students completed a two page, 35-item survey instrument. Results indicated that students who were regular exercisers (engaged in vigorous physical activity for 20 minutes $\geq 3$ days per week) were significantly more likely than students who were not regular exercisers to report higher levels of physical sexual satisfaction. Body mass index was also significantly correlated to physical sexual satisfaction. A series of multivariate analyses of variance revealed that physical sexual satisfaction and perceived impact of exercise on physical sexual satisfaction differed significantly based on gender. Overall, students perceived body image, sexual desirability perception, and physical strength as the three leading components of physical sexual satisfaction impacted by exercise. Since regular exercise may increase physical sexual satisfaction levels, health professionals could use the results of this study to help motivate individuals to enhance their physical sexual satisfaction by becoming more physically active.
\end{abstract}

(C) 2007 Californian Journal of Health Promotion. All rights reserved.

Keywords: exercise, sexuality, body image, college students

\section{Introduction}

In 2002, heart disease and stroke were the first and third leading causes of death in the United States killing over 800,000 Americans combined (National Center for Health Statistics [NCHS], 2004). These illnesses are the result of health conditions such as high blood pressure, high cholesterol, Type 2 diabetes, and obesity all of which can be reduced and prevented through regular physical activity (American College of Sports Medicine [ACSM], 2000). Currently in the United States, 100 million people are obese, 51 million have high cholesterol, 50 million have high blood pressure and 8 million have type 2 diabetes. These health conditions contribute to more frequent visits to the physician, more medication, more hospital visits, and higher health care expenditures. The annual cost of obesity alone is $\$ 117$ billion in the United States.
Physical activities have been studied in several laboratory-based research studies and have been documented to have various health benefits (ACSM, 2000). People who already have a chronic disease can enhance their ability to perform daily living activities through exercise (USDHHS, 2000a). Exercise can enable adults to improve and maintain strength and agility, decreasing the risk of falling, and thereby allow older adults to continue living independently and experiencing a higher quality of life. Along with these benefits, physiological benefits of exercise include: increased stroke volume, increased blood flow and circulation, increased aerobic capacity, decreased stress and depression, decreased risk of injury, decreased fatigue, reduced chance of dying prematurely, reduced chance of developing diabetes/high blood pressure/colon cancer, helps build and maintain 
health bones, muscles and joints, and assists in weight control (CDC, 1999).

While such health problems are highly preventable through regular physical activity, only $15 \%$ of individuals in the United States currently participate in the recommended weekly amount of physical activity (30 minutes of moderate physical activity for five or more days per week) and $40 \%$ participate in no leisure-time activity at all (USDHHS, 2000a). There have been a number of strategies used to motivate Americans to be physical active. Many of these strategies try to highlight all the benefits of physical activity. One of these strategies is educating individuals about the health benefits of physical activity. Another benefit mentioned in the literature is to increase physical sexual satisfaction. The research, however, conducted on this relationship has been scarce. The present study has been conducted to more fully explore this possible association.

Research studies that have examined the relationship between exercise and physical sexual satisfaction have all concluded that exercise increases physical sexual satisfaction levels (White, 1990). White found that the men who exercised regularly experienced enhanced sexual encounters including frequency of intimate activities, increased percentages of pleasing orgasms, and sufficient functioning during sex. A recent longitudinal study has found a positive correlation between exercise and sexual satisfaction in women experiencing menopause (Gerber, Johnson, Bunn, \& O’Brien, 2005). In addition, women who reported a decrease in frequency of exercise throughout the five year period also had a decrease in sexual satisfaction scores. Results of a study on females who began an exercise program revealed an increase in vaginal pulse amplitude and vaginal blood volume in both sexual functioning and sexually impaired women, suggesting that an increase in sympathetic nervous system arousal may produce the outcome of physiological responses in women (Meston \& Gorzalka, 1996). Exercise intensifies the sympathetic nervous system thus possibly enhancing physical sexual satisfaction. In a study conducted with cardiac male individuals, researchers concluded that the benefits of exercise training were correlated to improvements in sexual activity (Belardinelli, Lacalaprice, Faccenda, Purcaro, \& Perna, in press). Research involving active, older adults similarly found a correlation between physical sexual satisfaction and degree of fitness (Bortz \& Wallace, 1999).

The present study examines whether sexually active university students' perceived levels of physical sexual satisfaction differ based on exercise status, body mass index (BMI), and gender. In addition, it was studied whether perceived impact of exercise of physical sexual satisfaction differed based on gender and exercise status. Perceived impact of exercise on physical sexual satisfaction was defined for the purpose of this study as an individual's perception on the effects that exercise had on physical sexual satisfaction. There has been no study conducted that does a comparative analysis between exercise and physical sexual satisfaction. It should also be noted that the present study only examined components of physical sexual satisfaction, not emotional, since the physiology involved in exercise may influence physical aspects of sex but not emotional. If there was a more definite understanding of the association between exercise and physical sexual satisfaction then this may positively impact individual's to exercise.

\section{Methods}

\section{Participants}

The participants of this study consisted of students attending a Midwestern university during the Spring Quarter of $2005 \quad(n=678)$. Students were enrolled in general education courses taken by the general student population (i.e., Anatomy and Physiology, Physical Conditioning, Personal Behavior, Community Health, Racquetball, Yoga: Stress Management, and Group Fitness). Courses more likely to enroll regular exercisers were not targeted.

\section{Instrumentation}

A two page, 35-item survey instrument consisting of two subscales and a demographic segment was developed for this study. Subscale 
one assessed an individual's reported physical sexual satisfaction and required participants to indicate how satisfied or dissatisfied they were with each of the 18 items by using a five-point Likert-type scale $(1=$ strongly dissatisfied, $5=$ strongly satisfied). Individual item scores were totaled to achieve an overall sexual satisfaction score (potential range $=18-90$ ).

Subscale two assessed individuals' perceived impact of exercise on physical sexual satisfaction and required participants to indicate how strongly they agreed or disagreed with each of the 12 items by using a five-point Likert-type scale $(1=$ strongly disagree, $5=$ strongly agree $)$. All statements were worded in the positive direction, hence the higher the score, the higher the belief that exercise effects physical sexual satisfaction. For analysis purposes, individuals received a score of 5 for strongly agree, 4 for agree, 3 for neutral, 2 for disagree, and 1 for strongly disagree. Individual item scores were total 12 to 60 .

A panel of selected experts in health education and survey development and research were distributed the survey instrument to establish content validity. Successive adjustments were made in the instrument based on the recommendations offered from the panel of experts. Reliability and validity was determined for each of the subscales in the survey preceding the distribution of the survey for data collection. A test-retest design was used to determine the reliability of each subscale. A convenience sample of 20 individuals were distributed the survey during a Tuesday morning class. One week later, the same 20 individuals were redistributed the survey. Pearson correlation coefficients found stability reliability to be .749 for physical sexual satisfaction, .830 for exercise behaviors and .685 for perceived impact of exercise on physical sexual satisfaction. Internal consistency reliability analyses yielded Cronbach alphas of .914 for physical sexual satisfaction and .934 for perceptions on the effects of exercise on physical sexual satisfaction.

\section{Procedures}

After approval was granted from the institutional review board for human subjects, the study was undertaken. Permission to distribute the surveys was received from the instructors of eight Undergraduate level classes, a total of 17 classes. Arrangements were made with each individual instructor for convenient class days and time for the students to complete the survey. Students were informed of the research purpose and importance of the study through in class announcements made prior to survey distribution. Students were encouraged to complete the survey and reminded that it was voluntary and anonymous. The students were instructed to place their finished survey in a box placed in the front of the room face down. After each classroom completed the survey, the results were entered for data analysis.

\section{Data Analysis}

SPSS for Windows was used to analyze all data in the present study. A series of means, standard deviations, and frequency analyzes were conducted on demographic variables and individual subscale items. Pearson $r$ correlations were computed to determine whether physical sexual satisfaction significantly correlated with body mass index. Multivariate analyses of variance were used to determine whether reported physical sexual satisfaction and perceived impact of exercise on physical sexual satisfaction significantly differed based on current exercise status and gender.

\section{Results}

\section{Characteristics}

\section{Demographic and Background} Surveys were distributed to students $(n=678)$ at a Midwestern university in courses taken by the general student population. A total of 678 surveys were completed, resulting in an overall participation rate of $100 \%(678 / 678)$. Most students were female $(69.6 \%)$ and heterosexual $(97.6 \%)$ (Table 1). Nine in ten students were either single and not in a steady relationship or single and in a steady relationship with $85 \%$ of the steady relationships lasting longer that six months. Means for age, height, weight and body mass index is listed below in Table 1 . 
Table 1

Demographic and background characteristics of participants

\begin{tabular}{|c|c|c|}
\hline Demographic Characteristic (nonparametric variables) & $\mathbf{N}$ & Percent \\
\hline \multicolumn{3}{|l|}{ Gender } \\
\hline Female & 472 & 69.6 \\
\hline Male & 206 & 30.4 \\
\hline \multicolumn{3}{|l|}{ Relationship Status } \\
\hline Single \& Not in a Steady Relationship & 318 & 47.4 \\
\hline Single \& in a Steady Relationship & 286 & 42.4 \\
\hline Engaged & 29 & 4.3 \\
\hline Living with Partner & 29 & 4.3 \\
\hline Married & 12 & 1.8 \\
\hline \multicolumn{3}{|l|}{ Length of Relationship } \\
\hline Less than 6 months & 44 & 14.7 \\
\hline 6 months or Longer & 255 & 85.3 \\
\hline \multicolumn{3}{|l|}{ Sexual Orientation } \\
\hline Heterosexual & 657 & 97.6 \\
\hline Bisexual & 11 & 1.6 \\
\hline Homosexual & 5 & .7 \\
\hline Demographic Characteristics (parametric variables) & $\mathbf{M}$ & SD \\
\hline Age (Range $17-51$ years) & 20.67 & 3.1 \\
\hline Height (Range 53-78 inches) & 66.8 & 3.9 \\
\hline \multicolumn{3}{|l|}{ Weight } \\
\hline Range ( $88-275$ pounds) & 151.1 & 32.7 \\
\hline \multicolumn{3}{|l|}{ Body Mass Index } \\
\hline Range (15.14-39.24) & 23.7 & 4.1 \\
\hline
\end{tabular}

$\mathrm{N}=678 ; \%=$ Valid Percent; Missing values excluded from analyses

\section{Exercise Behavior}

Results indicated that students reported exercising an average of $3.03(\mathrm{SD}=1.850)$ days during an average week. On average, students reported that they exercised $2.82(\mathrm{SD}=1.968)$ days of the past seven days. Students who had partners reported that their partners exercised an average of $2.79(\mathrm{SD}=2.140)$ days during a typical week and that their partners exercised $2.61(\mathrm{SD}=2.192)$ days during the past 7 days. One in ten $(9.8 \%)$ students reported no participation in exercise during the past 6 months. Three in five $(62.1 \%)$ students exercised regularly, meaning that they exercised at least three days each week. Of those students, eight in ten $(78.6 \%)$ students participated in cardiovascular exercise, one in two (49.9\%) participated in weight training, and one in five
$(20.2 \%)$ in flexibility training. More specifically, $85 \%$ of the individuals who reported participating in flexibility training were female.

\section{Sexual Behavior}

Regarding sexual behavior, results indicated that one in five (20.1\%) students have never engaged in sexual activity. One in five (18.9\%) students reported engaging in sexual activity at some point in their life but not within the past 30 days. One in five $(21.4 \%)$ students also reported engaging in sexual activity within the past 30 days. Three in ten $(29.2 \%)$ students reported engaging in sexual activity within the past seven days. Of those students who reported engaging in sexual activity within the past seven days, an average of $2.13 \quad(\mathrm{SD}=1.816)$ days of sexual activity was reported. 


\section{Reported Physical Sexual Satisfaction}

Physical sexual satisfaction was rated on an 18 question, five point Likert-type scale that resulted in an overall physical sexual satisfaction score (1 point=extremely dissatisfied, 5 points=extremely satisfied). When comparing variables, results found that sexually active students were most satisfied with the frequency of their partner's orgasm $(\mathrm{M}=4.21, \mathrm{SD}=.764)$, partner's overall satisfaction $\quad(\mathrm{M}=4.13$,
$\mathrm{SD}=.767)$, and overall satisfaction $(\mathrm{M}=4.10$, $\mathrm{SD}=.735)$. Students reported being least satisfied with body image $(\mathrm{M}=3.18, \quad \mathrm{SD}=1.031)$, frequency $(\mathrm{M}=3.57, \mathrm{SD}=1.018)$, and partner's physical flexibility $(\mathrm{M}=3.75, \mathrm{SD}=.802)$. Physical sexual satisfaction scores ranged from 18 points (extremely dissatisfied sexual satisfaction) to 90 points (extremely sexual satisfied). The average score was $69.78(\mathrm{SD}=9.979)$ (Table 2).

Table 2

Participants' reported physical sexual satisfaction

\begin{tabular}{|l|c|c|}
\hline \multicolumn{1}{|c|}{ Physical Sexual Satisfaction Item } & Mean & SD \\
\hline Partner's Orgasm Frequency & 4.21 & .764 \\
\hline Partner's Overall Satisfaction & 4.13 & .767 \\
\hline Overall Satisfaction & 4.10 & .735 \\
\hline Energy & 4.04 & .725 \\
\hline Partner's Energy & 4.03 & .830 \\
\hline Ability to Move and Change Positions & 4.03 & .746 \\
\hline Partner's Ability to Move and Change Positions & 3.97 & .773 \\
\hline Partner's Physical Strength & 3.96 & .828 \\
\hline Sexually Desirability & 3.93 & .804 \\
\hline Physical Strength & 3.92 & .749 \\
\hline Self-Confidence & 3.88 & .806 \\
\hline Duration & 3.84 & .882 \\
\hline Orgasm Frequency & 3.83 & 1.049 \\
\hline Physical Flexibility & 3.81 & .883 \\
\hline Orgasm Intensity & 3.79 & .966 \\
\hline Partner's Physical Flexibility & 3.75 & .802 \\
\hline Frequency & 3.57 & 1.018 \\
\hline Body Image & 3.18 & 1.031 \\
\hline Physical Sexual Satisfaction Score & 69.78 & 9.979 \\
\hline
\end{tabular}

Means based on a five point scale (1=extremely dissatisfied, $5=$ =xtremely satisfied); Missing values excluded from analyses

\section{Reported Physical Sexual Satisfaction Based on Exercise Status, BMI, and Gender}

A multivariate analysis of variance (MANOVA) was conducted to examine the effect of exercise on physical sexual satisfaction. Results found that there was a significant difference between students who were sexually active and who exercised regularly and students who were sexually active and did not exercise regularly regarding physical sexual satisfaction $\mathrm{F}(18,509)=1.738, \mathrm{p}=.030$. More specifically, those who regularly exercised reported higher satisfaction regarding physical strength during sexual activity $(\mathrm{M}=3.97, \mathrm{SD}=.827)$ than nonexercisers $(\mathrm{M}=3.95, \mathrm{SD}=.827), \mathrm{F}(1,526)=$ $12.877 \mathrm{p}<.001$. Also, those who regularly exercised reported higher satisfaction regarding energy during sexual activity $(\mathrm{M}=4.07, \mathrm{SD}=$ 
.692) than those who did not exercise regularly $(\mathrm{M}=3.96, \mathrm{SD}=.778), \mathrm{F}(1,526)=6.744, \mathrm{p}=$ .010. Regular exercisers also reported higher satisfaction concerning duration of sexual activity $(\mathrm{M}=3.88, \mathrm{SD}=.885)$ than those who did not regularly exercise $(\mathrm{M}=3.78, \mathrm{SD}=$ $.942), \mathrm{F}(1,526)=5.632, \mathrm{p}=.018$. Those who exercised regularly also reported higher satisfaction concerning frequency of sexual activity $(\mathrm{M}=3.36 . \mathrm{SD}=1.009)$ than those who did not regularly exercise $(\mathrm{M}=3.49, \mathrm{SD}=$ $1.033), \mathrm{F}(1,526)=4.891, \mathrm{p}=.027$. In addition, those who exercised regularly reported higher overall satisfaction during sexual activity $(\mathrm{M}=$ $4.13, \mathrm{SD}=.715)$ than those who did not exercise regularly $(\mathrm{M}=4.04, \mathrm{SD}=.737), \mathrm{F}(1,526)=$ 4.634, $\mathrm{p}=.032$.

A bivariate correlation was conducted to examine the effect of body mass index on physical sexual satisfaction. Results showed that there was a significant correlation between students' physical sexual satisfaction and body mass index $(\mathrm{r}=.01, \mathrm{df}=531, \mathrm{p}=.019)$. As body mass index increased, students' physical sexual satisfaction decreased. A MANOVA was conducted to examine the effect of gender on physical sexual satisfaction. Results found that there was a significant difference between sexually active males who exercised regularly and sexually active females who exercised regularly regarding physical sexual satisfaction $\mathrm{F}(18,509)=7.949, \mathrm{p}<.001$. More specifically, sexually active females reported higher levels of sexual satisfaction regarding frequency of sexual activity $(\mathrm{M}=3.65, \mathrm{SD}=.952)$ than sexually active males $(\mathrm{M}=3.42, \mathrm{SD}=1.143), \mathrm{F}(1,526)=$ $8.605, \mathrm{p}=.003$, physical flexibility during sexual activity $(\mathrm{M}=3.87, \mathrm{SD}=.899)$ than sexually active males $(\mathrm{M}=3.70, \mathrm{SD}=.810)$, $\mathrm{F}(1,526)=4.728, \mathrm{p}=.030$, their partner's physical strength during sexual activity $(\mathrm{M}=4.11, \mathrm{SD}=.794)$ than sexually active males $(\mathrm{M}=3.64, \mathrm{SD}=.808), \mathrm{F}(1,526)=33.145, \mathrm{p}<$ .001 , their partner's ability to move and change positions during sexual activity $(\mathrm{M}=4.08, \mathrm{SD}=$ $.755)$ than sexually active males $(\mathrm{M}=3.75$, $\mathrm{SD}$ $=.765), \mathrm{F}(1,526)=24.183, \mathrm{p}<.001$, their partner's energy during sexual activity $(\mathrm{M}=$ $4.13, \mathrm{SD}=.805)$ than sexually active males $(\mathrm{M}$ $=3.82, \mathrm{SD}=.765), \mathrm{F}(1,526)=18.476, \mathrm{p}<.001$, their partner's overall satisfaction during sexually activity $(\mathrm{M}=4.17, \mathrm{SD}=.736)$ than sexually active males $(\mathrm{M}=4.00, \mathrm{SD}=.824)$, $\mathrm{F}(1,526)=7.532, \mathrm{p}=.006$, and their partner's orgasm frequency during sexually activity $(\mathrm{M}=$ $4.32, \mathrm{SD}=.705)$ than sexually active males $(\mathrm{M}$ $=3.95, \mathrm{SD}=.837), \mathrm{F}(1,526)=27.710, \mathrm{p}<.001$. Sexually active males reported higher satisfaction concerning body image during sexually activity $(\mathrm{M}=3.42, \mathrm{SD}=.909)$ than sexually active females $(\mathrm{M}=3.07, \mathrm{SD}=1.066)$, $\mathrm{F}(1,526)=7.073, \mathrm{p}=.008$, orgasm frequency during sexually activity $(\mathrm{M}=4.17, \mathrm{SD}=.775)$ than sexually active females $(\mathrm{M}=3.67, \mathrm{SD}=$ $1.122), F(1,526)=17.522, p<.001$, and orgasm intensity during sexually active $(\mathrm{M}=3.99, \mathrm{SD}=$ .707) than sexually active females $(\mathrm{M}=3.69$, $\mathrm{SD}=1.050), \mathrm{F}(1,526)=6.786, \mathrm{p}=.009$. It was therefore concluded that the gender of college students had an effect on physical sexual satisfaction. A MANOVA was also conducted to examine the combined effect of gender and regular exercise on physical sexual satisfaction. Results found no significant differences between males and females who exercised regularly regarding physical sexual satisfaction $\mathrm{F}(18,509)$ $=1.094, \mathrm{p}=.355$.

\section{Perceived Impact of Regular Exercise on Sexual Satisfaction}

Perceived impact of regular exercise on sexual satisfaction was rated on a 12 question,

five point Likert-type scale that gave each respondent an overall regular exercise belief score $(1$ point $=$ strongly disagree, 5 points $=$ strongly agree). When comparing variables, results found that students perceived body image $(\mathrm{M}=4.29, \quad \mathrm{SD}=.766), \quad$ sexual desirability perception $(\mathrm{M}=3.94, \mathrm{SD}=.839)$, and physical strength $(\mathrm{M}=3.82, \mathrm{SD}=.863)$ as the top three components of physical sexual satisfaction that are affected by exercise. Orgasm frequency $(\mathrm{M}=3.14, \mathrm{SD}=.914)$, orgasm intensity $(\mathrm{M}=3.16$, $\mathrm{SD}=.920)$, and frequency $(\mathrm{M}=3.25, \mathrm{SD}=1.050)$ were the lowest three components of sexual satisfaction affected by exercise. Regular exercise belief scores ranged from 12 points (strongly disagree) to 60 points (strongly agree). The average score was $43.04(\mathrm{SD}=8.600)$ (Table $3)$. 
Table 3

Participants' Perceived Impact of Exercise on Physical Sexual Satisfaction

\begin{tabular}{|l|c|c|}
\hline \multicolumn{1}{|c|}{ Physical Sexual Satisfaction Factors } & Mean & SD \\
\hline Body Image & 4.29 & .766 \\
\hline Sexual Desirability Perception & 3.94 & .839 \\
\hline Physical Strength & 3.82 & .863 \\
\hline Physical Flexibility & 3.80 & .877 \\
\hline Self-Confidence & 3.79 & .934 \\
\hline Energy & 3.75 & .882 \\
\hline Duration & 3.56 & .905 \\
\hline Partner's Overall Satisfaction Perception & 3.53 & .911 \\
\hline Partner's Orgasm Frequency & 3.25 & .915 \\
\hline Frequency & 3.25 & 1.050 \\
\hline Orgasm Intensity & 3.16 & .920 \\
\hline Orgasm Frequency & 3.14 & .914 \\
\hline Effect of Exercise on Physical Sexual Satisfaction Score & 43.04 & 8.600 \\
\hline
\end{tabular}

Means based on a five point scale ( $1=$ strongly disagree, $5=$ strongly agree); Missing values excluded from analyses

Impact of Exercise on Physical Sexual Satisfaction Based on Exercise Status

A MANOVA conducted to examine the effect of regular exercise on perceived impact of exercise on physical sexual satisfaction indicated that students who exercised regularly differed significantly from students who did not exercise regularly regarding perceived impact of exercise on physical sexual satisfaction, $\mathrm{F}(12,646)=$ $1.735, \mathrm{p}=.05$. Univariate analyses conducted on individual physical sexual satisfaction items revealed additional significant differences based on exercise status (Table 4).

Table 4

Reported Physical Sexual Satisfaction Based on Exercise Status

\begin{tabular}{|l|c|c|c|c|c|r|}
\hline \multirow{2}{*}{ Physical Sexual Satisfaction Item } & \multicolumn{2}{|c|}{$\begin{array}{c}\text { Regular } \\
\text { Exerciser }^{\mathbf{a}}\end{array}$} & \multicolumn{2}{|c|}{ Non-Exerciser $^{\mathbf{b}}$} & \multirow{2}{*}{ F } & \multirow{2}{*}{ p } \\
\cline { 2 - 5 } & $\mathbf{M}$ & SD & M & SD & & \\
\hline Physical Strength & 3.99 & .739 & 3.78 & .757 & 12.877 & $<.001$ \\
\hline Energy & 4.07 & .692 & 3.96 & .778 & 6.744 & .010 \\
\hline Duration & 3.88 & .835 & 3.78 & .942 & 5.632 & .018 \\
\hline Frequency & 3.63 & 1.009 & 3.49 & 1.033 & 4.891 & .027 \\
\hline Overall Satisfaction & 4.13 & .715 & 4.04 & .737 & 4.634 & .032 \\
\hline
\end{tabular}

Means based on a five point scale ( $1=$ extremely dissatisfied, $5=$ extremely satisfied). Only significant differences shown.

${ }^{a}$ Regular exerciser defined as an individual that participates in physical activity 3 or more times per week, at least 20 minutes each session that makes you sweat or breathe hard.

${ }^{\mathrm{b}}$ Non-exerciser defined as an individual that does not participate in physical activity 3 or more times per week, at least 20 minutes each session that makes you sweat or breathe hard. 


\section{Impact of Exercise on Physical Sexual Satisfaction Based on Gender}

Regarding effect of gender on perceived impact of exercise on physical sexual satisfaction, results found that there was a significant difference between males and females regarding perceived impact of exercise on physical sexual satisfaction, $F(18,646)=2.899, p=.001$. More specifically, females reported higher levels of perceived impact of exercise on physical sexual satisfaction regarding body image during sexual activity $(\mathrm{M}=4.34, \mathrm{SD}=.731)$ than males $(\mathrm{M}=$ $4.18, \mathrm{SD}=.825), \mathrm{F}(1,657)=9.721, \mathrm{p}=.002$. Males reported higher levels of perceived impact of exercise on physical sexual satisfaction regarding their partner's orgasm frequency during sexually activity $(\mathrm{M}=3.60, \mathrm{SD}=.867)$ than females $(\mathrm{M}=3.49, \mathrm{SD}=.930), \mathrm{F}(1,657)=$ $5.716, \mathrm{p}=.017$ (Table 5).

Table 5

Reported Physical Sexual Satisfaction Based on Gender

\begin{tabular}{|c|c|c|c|c|c|c|}
\hline \multirow{3}{*}{\begin{tabular}{|c|} 
Physical Sexual Satisfaction Item \\
Partner's Ability to Move and Change Positions
\end{tabular}} & \multicolumn{2}{|c|}{ Male } & \multicolumn{2}{|c|}{ Female } & \multirow{2}{*}{$\mathbf{F}$} & \multirow{3}{*}{$\begin{array}{c}\mathbf{p} \\
<.001\end{array}$} \\
\hline & $\mathbf{M}$ & SD & $\mathbf{M}$ & SD & & \\
\hline & 3.75 & .765 & 4.08 & .755 & 24.183 & \\
\hline Partner's Energy & 3.82 & .841 & 4.13 & .805 & 18.476 & $<.001$ \\
\hline Partner's Physical Strength & 3.64 & .808 & 4.11 & .794 & 33.145 & $<<.001$ \\
\hline Partner's Orgasm Frequency & 3.95 & .837 & 4.32 & .705 & 27.710 & $<<.001$ \\
\hline Orgasm Frequency & 4.17 & .775 & 3.67 & 1.122 & 17.522 & $<.001$ \\
\hline Frequency & 3.42 & 1.143 & 3.65 & .952 & 8.605 & .003 \\
\hline Physical Flexibility & 3.70 & .810 & .387 & .899 & 4.728 & .030 \\
\hline Partner's Overall Satisfaction & 4.00 & .824 & 4.17 & .736 & 7.532 & .006 \\
\hline Body Image & 3.42 & .909 & 3.07 & 1.066 & 7.073 & .008 \\
\hline Orgasm Intensity & 3.99 & .707 & 3.69 & 1.050 & 6.786 & .009 \\
\hline
\end{tabular}

Means based on a five point scale (1=extremely dissatisfied, $5=$ extremely satisfied); Only significant differences shown

A MANOVA was conducted to examine the combined effect of regular exercise and gender on perceived impact of exercise on physical sexual satisfaction. Results found no significant difference between males and females who exercised regularly regarding perceived sexual satisfaction, $\mathrm{F}(12,646)=1.366, \mathrm{p}=.177$ (Table $6)$.

Table 6

Perceived impact of exercise on physical sexual satisfaction based on gender

\begin{tabular}{|c|c|c|c|c|c|c|}
\hline \multirow{2}{*}{ Physical Sexual Satisfaction Item } & \multicolumn{2}{|c|}{ Male } & \multicolumn{2}{|c|}{ Female } & \multirow{2}{*}{$\mathbf{F}$} & \multirow[b]{2}{*}{$\mathbf{p}$} \\
\hline & & SD & & SD & & \\
\hline Body Image & 4.18 & .825 & 4.34 & .731 & 9.721 & .002 \\
\hline Partner's Orgasm Frequency & 3.38 & .947 & 3.18 & .891 & 5.716 & .017 \\
\hline
\end{tabular}




\section{Discussion}

Despite the various efforts to educate Americans on the health benefits of exercise, only $15 \%$ of individuals living in the United States participate in the recommended amount of physical activity (30 minutes of moderate physical activity, three or more days per week) and $40 \%$ participate in no physical activity. The United States Department of Health and Human Services (2000b) reported that one in four adults who have an advanced degree in education engage in a high level of physical activity. The present study found that three in five college students working towards an advanced degree, were considered to exercise regularly (three or more times per week for at least 20 minutes each session). This may be because the participants consisted of college students. A recent study found that only $29 \%$ of college students reported not exercising (Racette, Deusinger, Strube, Highstein, \& Deusinger, 2005).

Sexual behaviors among college students in the present study indicated that $29 \%$ engaged in sexual activity within the past seven days, $21 \%$ within the past 30 days, $19 \%$ at some point in their life but not within the past 30 days, and $20 \%$ had never engaged in sexual activity. Such findings parallel closely to those found by Ratliff-Crain et Al. (1999) in which $65 \%$ of college students had engaged in sexual intercourse at least once in their life.

The present study found a significant relationship between physical sexual satisfaction and regular exercise in college students. This relationship was also found in past studies involving males (White et al., 1990; Belardinelli et al., in press), females (Gerber et al., 2005; Meston \& Gorzalka, 1996), older adults (Bortz \& Wallace, 1999), athletes (Whitten, 1994), and sexually dysfunctional individuals (Belardinelli et al., in press, Meston \& Gorzalka, 1996; White et al., 1990). Regular exercisers may experience physiological benefits that could enhance their physical sexual satisfaction such as increased blood flow, joint and muscular flexibility, release of endorphins, and skeletal muscular strength. A past study suggested that through increased skeletal muscle tone, orgasmic pleasure could be increased (Mandall, 1979).
Psychological benefits of exercise such as decreased depression or stress (Ensel \& Lin, 2004) could also impact an individual's physical sexual satisfaction. Therefore, if an individual is seeking to increase his/her physical sexual satisfaction, a possible solution could be to exercise regularly. Also, health educators will now have an additional incentive to motive individuals to get involve in an exercise program. Since it is concluded from the abundant sales of sexual materials in American that most individuals want active, satisfying sex lives, knowing that exercise can help enhance their sex lives may lead more people to actually exercise.

While no previous study has found a difference between male and female levels of physical sexual satisfaction, the present study has found a relationship between gender and physical sexual satisfaction. Males reported higher levels of physical sexual satisfaction regarding orgasm frequency, body image, and orgasm intensity. This may indicate males have more selfconfidence and therefore a better body image or that males are not as affected by society's push to have a particular body image as many females may be. It may also be noted that orgasmic females are often unable to reach climax due to premature ejaculation by the male and the refractory period (Wikipedia Encyclopedia, 2005). Therefore, it may be concluded that males are more satisfied with their orgasm frequency because they achieve an orgasm more often than females. According to a study that examined gender differences in sexual intercourse experiences among college students, males were more likely to have an orgasm than females (Sprecher, Barbee, \& Schwartz, 1995).

Females in the present study reported higher levels of physical sexual satisfaction regarding partner's ability to move and change positions during sexual activity, partner's energy during sexual activity, partner's physical strength during sexual activity, partner's orgasm frequency, frequency of sexual activity, physical flexibility during sexual activity, and partner's overall satisfaction during sexual activity. This may indicate that females are satisfied with their partner's role during sexual activity but not 
satisfied with their own performance during sexual activity. Performance anxiety is believed to be the most common cause of orgasm problems (Medline Plus Medical Encyclopedia, 2004). The one area that females reported higher levels of physical sexual satisfaction involving their own bodies was physical flexibility during sexual activity. Results of the present study indicate that of the $85 \%$ of the individuals that reported participating in flexibility training were female. Since the specific components that lead to increased satisfaction are different between males and females, couples should talk to one another as a means to meet each other's sexual needs and desires.

The present study also found a correlation between body mass index and physical sexual satisfaction. The correlation determined that as body mass index increases, physical sexual satisfaction decreases and as body mass index decreases, physical sexual satisfaction increases. Supporting this finding, a previous study found that individuals with a lower or average body mass index had higher levels of selfattractiveness (Haavio-Mannila \& Purhonen, 2001). This may indicate that individuals with a lower or average body mass index feel more attractive and therefore have increased levels of sexual satisfaction. White et al. (1990) found that individuals reported being more satisfied with their physical appearance and "feeling of well-being." The study also showed that a decrease in body fat was consistent with changes in self-perception of physical body image which might suggest changes in feelings towards sexuality and interest from a sexual partner. Gerber et al. (2005) also found a positive correlation between an individual's body image and sexual satisfaction. An individual's body image could be impacted by their body mass index. Therefore, individuals that are interested in possibly enhancing their sexual satisfaction should consider maintaining a low or average body mass index. The use of regular exercise may assist in meeting this goal.

Sexual satisfaction levels are affected not only from an individual's physiology or physical make-up but also from emotional state of the individual/relationship, societal impact, and psychological factors. The current study examined sexual satisfaction from a physical stand point only. It may be noted that sexual satisfaction cannot be solely determined through physical measures but would require a complete examination of all components impacting sexuality.

Finally, the limitations of this study should be noted. Such limitations included participants' willingness to report their answers honestly, participants' belief that their responses would be kept confidential, and participants' comfort level of responding to sensitive items regarding sexual behavior and physical sexual satisfaction. In addition, since the sample comprised students from one Midwestern university, caution should be taken when attempting to generalize the findings of this study.

\section{Recommendations}

Based on the findings of this study several recommendations are offered. Health educators could educate individuals on the potential positive effects of regular exercise and low BMI levels on physical sexual satisfaction. Becoming more aware of the potential benefits of regular exercise and decreased body mass index may help to motivate some individuals to begin engaging in regular physical activity and participate in behaviors that decrease their body mass index (e.g., healthy diet and exercise). Additional research is needed to more thoroughly explore the effect of physical activity on sexual satisfaction. Future research should examine individuals in a controlled environment, similar to the previous research (Gerber et al., 2005; Meston \& Gorzalka, 1996; White et al., 1990). A case-control study is greatly needed. Such a study could consist of two experimental conditions. The first group would consist of sexually active couples who do not exercise during the entire course of the study. The second group would consist of sexually active couples who do not exercise at the beginning of the study but are taught to exercise during the study. Both groups would be surveyed regarding their physical sexual satisfaction at pretest and posttest to determine any potential differences. In this manner, possible cause-and-effect results could be 
determined. Finally, future studies should examine additional factors, affecting physical sexual satisfaction such as how exercise impacts emotional sexual satisfaction. Do partners who exercise together feel more emotionally close and do they report higher levels of overall sexual satisfaction? Much research is clearly warranted regarding this issue.

\section{References}

American College of Sports Medicine [ACSM]. (2000). ACSM's guidelines for exercise testing and prescription (6th ed.). Philadelphia: Lippincott Williams \& Wilkins.

Belardinelli, R., Lacalaprice, F., Faccenda, E., Purcaro, A., \& Perna, G. (in press). Effects of short-term moderate exercise training on sexual function in male patients with chronic stable heart failure. International Journal of Cardiology.

Bortz, W., \& Wallace, D. (1999). Physical fitness, aging, and sexuality. The Western Journal of Medicine, 170(3), 167-168.

Center for Disease Control and Prevention [CDC]. (1999). The link between physical activity and morbidity and mortality. Retrieved on January 16, 2005, from http://cdc.gov/nccdphp/sgr/mm.htm

Center for Disease Control and Prevention [CDC]. (2004b). How can I overcome barriers to physical activity? Retrieved on February 15, 2005, from http://cdc.gov/nccdphp/dnpa/physical/life/overcome.htm

Ensel, W., \& Lin, N. (2004). Physical fitness and the stress process. Journal of Community Psychology 32(1), 81-101.

Gerber, J., Johnson, J., Bunn, J., \& O'Brien, S. (2005). A longitudinal study of the effects of free testosterone and other psychosocial variables on sexual function during the natural traverse of menopause. Fertility and Sterility, 83, 643-648.

Greenberg, J., Bruess, C., \& Haffner, D. (2004). Exploring the dimensions of human sexuality (2nd ed.). Sudbury, MA: Jones and Bartlett Publishing.

Haavio-Mannila, E, \& Purhonen, S. (2001). Slimness and self-related sexual attractiveness: comparisons among men and women of two cultures. Journal of Sex Research, 38(2), 102-111.

Mandall, A. (1979). The second wind. Psychiatric Annals, 9, 153-160.

Medline Plus Medical Encyclopedia. (2004). Orgasmic dysfunction. Retrieved on July 7, 2005, from http://www.nlm.nih.gov/medlineplus/ency/article/001953.htm

Meston, C., \& Gorzalka, B. (1996). Differential effects of sympathetic activation on sexual arousal in sexually dysfunctional and functional women. Journal of Abnormal Psychology, 105, 585-591.

National Center for Health Statistics [NCHS]. (2004). Fast stats. Retrieved on February 13, 2005, from http://www.cdc.gov/nchs/fastats/deaths.htm

Racette, S., Deusinger, S., Strube, M., Highstein, G., \& Deusinger, R. (2005). Weight changes, exercise, and dietary patterns during freshman and sophomore years of college. Journal of American College Health, 53, 245-252.

Sexuality drug market makes inroad. (2004). Contemporary Sexuality, 38(1), 15.

Sprecher, S., Barbee, A., \& Schwartz, P. (1995). "Was it good for you, too? Gender differences in first sexual intercourse experiences. Journal of Sex Research, 32(1), 3-16.

United States Department of Health and Human Services. (2000b). Physical activity among adults: United States, 2000. Retrieved December 1, 2007, from http://www.fitness.gov/activity levels.htm

United States Department of Health and Human Services. (2000a). Healthy People 2010. With understanding and improving health and objectives for improving health (2 Vol.). Washington, D.C.: Government Printing Office.

White, J., Case, D., McWhirter, D., \& Mattison, A. (1990). Enhanced sexual behavior in exercising men. Archives of Sexual Behavior, 19, 193-209.

Whitten, P. (1994). The complete book of swimming. New York: Random House Inc.

Wikipedia Encyclopedia. (2005). Orgasm. Retrieved on July 7, 2005, from http://en.wikipedia.org/wiki/Orgasm 
Author Information

Holly C. Lindeman, MEd

Graduate Assistant

University of Cincinnati

Cincinnati, OH 45221-0068

Ph.: 706/534-8691

E-Mail: HCLindeman@aol.com

Keith A. King, PhD, CHES*

Associate Professor of Health Promotion

University of Cincinnati

Cincinnati, OH 45221-0068

Ph.: 513-556-3859

E-Mail: keith.king@uc.edu

Bradley R. Wilson, $\mathrm{PhD}$

Professor of Health Promotion

University of Cincinnati

Cincinnati, OH 45221-0068

* corresponding author 